OPEN ACCESS

Edited by:

Marian Brestic,

Slovak University of Agriculture,

Slovakia

Reviewed by:

Xiangnan $\mathrm{Li}$,

Northeast Institute of Geography and Agroecology (CAS), China

Xinghong Yang,

Shandong Agricultural University,

China

Lorenzo Ferroni,

University of Ferrara, Italy

*Correspondence:

Shi-Bao Zhang

sbzhang@mail.kib.ac.cn

Tao Liu

52133490@qq.com

Wei Huang

huangwei@mail.kib.ac.cn

Specialty section: This article was submitted to

Plant Abiotic Stress,

a section of the journal

Frontiers in Plant Science

Received: 10 January 2018 Accepted: 09 February 2018 Published: 26 February 2018

Citation: Huang W, Yang Y-J, Zhang S-B and

Liu T (2018) Cyclic Electron Flow around Photosystem I Promotes ATP Synthesis Possibly Helping the Rapid Repair of Photodamaged Photosystem II at Low Light.

Front. Plant Sci. 9:239. doi: 10.3389/fpls.2018.00239

\section{Cyclic Electron Flow around Photosystem I Promotes ATP Synthesis Possibly Helping the Rapid Repair of Photodamaged Photosystem II at Low Light}

\author{
Wei Huang ${ }^{1 *}$, Ying-Jie Yang ${ }^{1,2}$, Shi-Bao Zhang ${ }^{1 *}$ and Tao Liu ${ }^{3 *}$ \\ ${ }^{1}$ Key Laboratory of Economic Plants and Biotechnology, Kunming Institute of Botany, Chinese Academy of Sciences, \\ Kunming, China, ${ }^{2}$ University of Chinese Academy of Sciences, Beijing, China, ${ }^{3}$ National-Local Joint Engineering Research \\ Center on Germplasm Utilization and Innovation of Chinese Medicinal Materials in Southwest China, Yunnan Agricultural \\ University, Kunming, China
}

In higher plants, moderate photoinhibition of photosystem II (PSII) leads to a stimulation of cyclic electron flow (CEF) at low light, which is accompanied by an increase in the P700 oxidation ratio. However, the specific role of CEF stimulation at low light is not well known. Furthermore, the mechanism underlying this increase in P700 oxidation ratio at low light is unclear. To address these questions, intact leaves of the shadeadapted plant Panax notoginseng were treated at $2258 \mu \mathrm{mol}$ photons $\mathrm{m}^{-2} \mathrm{~s}^{-1}$ for 30 min to induce PSII photoinhibition. Before and after this high-light treatment, PSI and PSII activity, the energy quenching in PSII, the redox state of PSI and proton motive force (pmf) at a low light of $54 \mu \mathrm{mol}$ photons $\mathrm{m}^{-2} \mathrm{~s}^{-1}$ were determined the steady state. After high-light treatment, electron flow through PSII (ETRII) significantly decreased but CEF was remarkably stimulated. The P700 oxidation ratio significantly increased but non-photochemical quenching changed negligibly. Concomitantly, the total pmf decreased significantly and the proton gradient $(\Delta \mathrm{pH})$ across the thylakoid membrane remained stable. Furthermore, the P700 oxidation ratio was negatively correlated with the value of ETRII. These results suggest that upon PSII photoinhibition, CEF is stimulated to increase the ATP synthesis, facilitating the rapid repair of photodamaged PSII. The increase in P700 oxidation ratio at low light cannot be explained by the change in pmf, but is primarily controlled by electron transfer from PSII.

Keywords: ATP synthesis, cyclic electron flow, linear electron flow, proton motive force, PSII photoinhibition, P700 oxidation ratio

\section{INTRODUCTION}

Although light is the driving force of photosynthesis, high light can cause significant photoinhibition of photosystem II (PSII) for leaves of shade-establishing plants (Kitao et al., 2000; Barth et al., 2001; Krause et al., 2004; Huang et al., 2015b, 2016b,c). However, photoinhibited PSII recovers rapidly at low light (He and Chow, 2003; Zhang and Scheller, 2004, Allakhverdiev et al., 2005), due to the fast turnover rate of D1 protein (Aro et al., 1993; Zhang and Scheller, 2004; 
Allakhverdiev et al., 2005). The rate of PSII repair was reduced upon inhibition of the synthesis of ATP either via PSI or PSII (Allakhverdiev et al., 2005), indicating that the fast repair of photodamaged PSII complexes needs a large amount of ATP in a short time. A previous study indicated that after chillinginduced photoinhibition of PSII, cyclic electron flow (CEF) was significantly stimulated during recovery at low light (Huang et al., 2010). It was proposed that this stimulation of CEF at low light enhanced the synthesis of ATP for the fast repair of PSII (Huang et al., 2010). However, more evidence is needed to clarify the specific role of CEF stimulation at low light after PSII photoinhibition.

During CEF, electrons from either NADPH or ferredoxin are cycled around PSI into the plastoquinone pool. This electron transfer is coupled to proton translocation and generates a proton gradient across thylakoid membranes $(\Delta \mathrm{pH})$ (Shikanai and Yamamoto, 2017). In addition to contributing ATP synthesis, another function of $\Delta \mathrm{pH}$ is the down-regulation of photosynthetic electron transport by acidifying the thylakoid lumen (Shikanai, 2014, 2016). This regulation involves two different mechanisms: one is linked to thermal energy dissipation and dissipates excess absorbed light energy as heat from PSII antennae (Takahashi et al., 2009), and the other one is downregulation of Cyt $b_{6} / f$ complex activity and controls the rate of electron transfer to PSI (Suorsa et al., 2012, 2016; Tikkanen and Aro, 2014). In angiosperms including Arabidopsis thaliana, two pathways of PSI cyclic electron transport are operating (Shikanai, 2007). The first CEF pathway is PGR5-/PGRL1dependent pathway sensitive to antimycin A (Munekage et al., 2002; Sugimoto et al., 2013), and the other one is dependent on the chloroplast NADH dehydrogenase-like (NDH) complex (Burrows et al., 1998; Shikanai et al., 1998). The contribution of the PGR5-/PGRL1-dependent pathway is more significant in C3 plants. Under high light, the activation of PGR5-/PGRL1dependent CEF induces the acidification of thylakoid lumen and thus leads to the high levels of P700 oxidation ratio (Suorsa et al., 2012, 2016; Kono et al., 2014; Yamori et al., 2016). By comparison, the P700 oxidation ratio at low light was little affected by the deficiency in PGR5-/PGRL1-dependent CEF (Munekage et al., 2002, 2004; Kono et al., 2014). Upon moderate PSII photoinhibition, the stimulation of CEF at low light was accompanied with an increase in the P700 oxidation ration (Huang et al., 2010). However, it is unclear whether this increased P700 oxidation ratio is caused by the CEF stimulation.

In addition to CEF, electron transfer from PSII (ETRII) plays an important role in affecting the redox state of PSI (Tikkanen et al., 2014; Huang et al., 2016a,c; Suorsa et al., 2016). In pgr5 plants of $A$. thaliana, severe photoinhibition of PSII is likely to function as the ultimate control of photosynthetic electron transfer allowing the maintenance of P700 optimally oxidized under excess light (Tikkanen et al., 2014). Recently, Suorsa et al. (2016) indicated that pgr5 plants showed high P700 oxidation ratio under high light when the ETRII was reduced by combining knockout mutations in PsbO1, PsbP2, $P s b Q 1, P s b Q 2$, and $P s b R$ loci. In chilled leaves of tobacco, moderate PSII photoinhibition depressed electron flow to PSI and then increased the P700 oxidation ratio during further chilling treatments (Huang et al., 2016a). Furthermore, chillinginduced photoinhibition of PSII led to a depression of ETRII, which was accompanied with an increase in the P700 oxidation ratio at low light (Huang et al., 2010). We hypothesize that the P700 oxidation ratio at low light is primarily controlled by electron flow from PSII.

In our previous report, we observed that the shadeestablishing plant Panax notoginseng showed selective photoinhibition of PSII under high-light stress. After short-term exposure to high-light stress, ETRII at low light significantly decreased but CEF was significantly stimulated. Concomitantly, the P700 oxidation ratio largely increased. Our specific objectives were to (1) investigate whether CEF stimulation at low light mainly facilitates the synthesis of ATP; and (2) determine whether the increase in $\mathrm{P} 700$ oxidation ratio upon moderate PSII photoinhibition is more related to ETRII rather than the CEF stimulation or the change in pmf. In order to address these questions, intact leaves of Panax notoginseng were treated at $2258 \mu \mathrm{mol}$ photons $\mathrm{m}^{-2} \mathrm{~s}^{-1}$ for $30 \mathrm{~min}$. Before and after high-light treatment, the energy distribution in PSII, the redox state of PSI and proton motive force $(p m f)$ at a low light of $54 \mu \mathrm{mol}$ photons $\mathrm{m}^{-2} \mathrm{~s}^{-1}$ were determined.

\section{MATERIALS AND METHODS}

\section{Plant Materials and Growth Condition}

In the present study, 2-years-old plants of a shade-establishing plant Panax notoginseng (Burkill) F. H. Chen ex C. Chow and W. G. Huang were used for experiments. These plants were grown at light condition of $10 \%$ sunlight (with maximum midday light intensity of approximately $200 \mu \mathrm{mol}$ photons $\mathrm{m}^{-2} \mathrm{~s}^{-1}$ ). No water and nutrition stresses were experienced for these plants. 9-weeks-old fully expanded leaves were used for the photosynthetic measurements.

\section{PSI and PSII Measurements}

Photosystem I and PSII parameters were measured at $25^{\circ} \mathrm{C}$ by simultaneously recorded using a Dual PAM-100 measuring system (Heinz Walz GmbH, Effeltrich, Germany). The chlorophyll fluorescence parameters were calculated as follows: $F_{\mathrm{v}} / F_{\mathrm{m}}=\left(F_{\mathrm{m}}-F_{\mathrm{o}}\right) / F_{\mathrm{m}}, Y(\mathrm{II})=\left(F_{\mathrm{m}}^{\prime}-F_{\mathrm{s}}\right) / F_{\mathrm{m}}^{\prime} \quad$ (Genty et al., 1989), $\mathrm{NPQ}=\left(F_{\mathrm{m}}-F_{\mathrm{m}}^{\prime}\right) / F_{\mathrm{m}}^{\prime} . F_{\mathrm{o}}$ and $F_{\mathrm{m}}$ are the minimum and maximum fluorescence after dark acclimation, respectively. $F_{\mathrm{s}}$ is the light-adapted steady-state fluorescence. $F_{\mathrm{o}}$ and $F_{\mathrm{m}}$ were determined after dark acclimation for $30 \mathrm{~min}$ before and after high-light treatment. The PSI photosynthetic parameters were measured by Dual PAM-100 based on P700 signal (difference of intensities of 830 and $875 \mathrm{~nm}$ pulsemodulated measuring light reaching the photodetector) (Klughammer and Schreiber, 2008). The P700 ${ }^{+}$signals $(P)$ may vary between a minimal (P700 fully reduced) and a maximal level (P700 fully oxidized). The maximum level $\left(P_{\mathrm{m}}\right)$ was determined with application of a saturation pulse $(600 \mathrm{~ms}$ and $10000 \mu \mathrm{mol}$ photons $\mathrm{m}^{-2} \mathrm{~s}^{-1}$ ) after pre-illumination with far-red light, and $P_{\mathrm{m}}$ was used to estimate the PSI activity. $P_{\mathrm{m}}{ }^{\prime}$ was determined similar to $P_{\mathrm{m}}$ but with actinic light instead of far-red light. 
The quantum yield of PSI was calculated as $\mathrm{Y}(\mathrm{I})=$ $\left(P_{\mathrm{m}}^{\prime}-P\right) / P_{\mathrm{m}}$. The $\mathrm{P} 700$ oxidation ratio in a given actinic light was calculated as $\mathrm{Y}(\mathrm{ND})=P / P_{\mathrm{m}}$. The quantum yield of PSI non-photochemical energy dissipation due to acceptor-side limitation was calculated as $\mathrm{Y}(\mathrm{NA})=\left(P_{\mathrm{m}}-P_{\mathrm{m}}^{\prime}\right) / P_{\mathrm{m}}$. The steady state values for $\mathrm{Y}(\mathrm{II}), \mathrm{NPQ}, \mathrm{Y}(\mathrm{I})$, and $\mathrm{Y}(\mathrm{ND})$ were measured after light acclimation at low light for $20 \mathrm{~min}$.

Photosynthetic electron flow through PSI and PSII were calculated as: $\mathrm{ETRII}=Y(\mathrm{II}) \times P P F D \times 0.84 \times 0.5($ Krall and Edwards, 1992), ETRI $=Y(\mathrm{I}) \times P P F D \times 0.84 \times 0.5$ (Yamori et al., 2011), where 0.5 is assumed to be the proportion of absorbed light reaching PSI or PSII, and 0.84 is assumed to be the absorptance (the fraction of the incident light absorbed by leaves). The extent of CEF activation was estimated as ETRI/ETRII ratio (Yamori et al., 2011, 2015). It should be noted that the 1:1 excitation partitioning between PSI and PSII may be not real, because shade plants tend to increase the PSII/PSI ratio (Lunde et al., 2003; Tikkanen et al., 2006; Grieco et al., 2012). Furthermore, in this study, PSI and PSII parameters were measured using red actinic light $(635 \mathrm{~nm})$ provided by DualPAM 100 (Walz, Germany). Because red actinic light favors the excitation of PSII over that of PSI, the excitation energy distribution from LHCII to PSII and PSI may be affected by the red actinic light (Tikkanen et al., 2017).

\section{Electrochromic Shift Analysis}

The ECS signal was monitored as the absorbance change at 515 nm using a Dual-PAM-100 (Walz, Effeltrich, Germany) equipped with a P515/535 emitter-detector module (Walz). The ECS signal was obtained after $20 \mathrm{~min}$ of illumination at $54 \mu \mathrm{mol}$ photons $\mathrm{m}^{-2} \mathrm{~s}^{-1}$, afterwards, the ECS decay was measured by switching off the actinic light for $30 \mathrm{~s}$. The analysis of

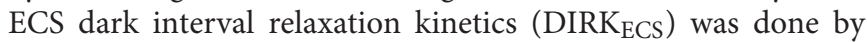
the method of Sacksteder et al. (2001) and Takizawa et al. (2008). Total pmf was estimated from the total amplitude of the rapid decay of the ECS signal during $300 \mathrm{~ms}$ dark interval. The slow relaxation of ECS signal enabled to recognize the contribution of proton gradient across the thylakoid membranes $(\Delta \mathrm{pH})$. The time constant of the first-order ECS relaxation $\left(\tau_{\mathrm{ECS}}\right)$ is inversely proportional to the proton conductivity $\left(g_{\mathrm{H}}{ }^{+}\right)$of the thylakoid membrane through the ATP synthase (Sacksteder and Kramer, 2000; Cruz et al., 2005). As a result, $g_{\mathrm{H}}{ }^{+}$was estimated as the inverse of the decay time constant $\left[1 / \tau_{\mathrm{ECS}}\right]$.

\section{Photoinhibitory Treatments}

After dark acclimation for $30 \mathrm{~min}, F_{\mathrm{v}} / F_{\mathrm{m}}$ and $P_{\mathrm{m}}$ were measured in intact leaves. Afterwards, these intact leaves were light-adapted at $59 \mu \mathrm{mol}$ photons $\mathrm{m}^{-2} \mathrm{~s}^{-1}$ for $20 \mathrm{~min}$ and parameters of chlorophyll fluorescence, P700 signal and ECS signal were recorded. Then the actinic light was changed to $2258 \mu \mathrm{mol}$ photons $\mathrm{m}^{-2} \mathrm{~s}^{-1}$. After exposure to this high light for $30 \mathrm{~min}$, the actinic light was changed to $59 \mu \mathrm{mol}$ photons $\mathrm{m}^{-2} \mathrm{~s}^{-1}$ immediately and the photosynthetic parameters were recorded after light acclimation for $20 \mathrm{~min}$. Finally, $F_{\mathrm{v}} / F_{\mathrm{m}}$ and $P_{\mathrm{m}}$ were measured after dark acclimation for $30 \mathrm{~min}$.

\section{Statistical Analysis}

The results were displayed as mean values of five independent experiments. Independent $T$-test was used at $\alpha=0.05$ significance level to determine whether significant differences existed between different treatments. All statistical analyses were performed using SPSS 16.0.

\section{RESULTS}

During photosynthetic induction at $59 \mu \mathrm{mol}$ photons $\mathrm{m}^{-2} \mathrm{~s}^{-1}$, ETRII gradually increased and reached a steady state at about 18 min (Figure 1A). By comparison, ETRI was constant during this induction phase (Figure 1A). After onset of this low light, ETRI was much higher than ETRII. However, after a 20-min photosynthetic induction, ETRI was lower than ETRII. These results suggested the activation of CEF during the initial phase of induction, in accordance with previous studies (Joliot and Joliot, 2002, 2005; Makino et al., 2002). After this photosynthetic induction at low light, leaves were illuminated at a high light of $2258 \mu \mathrm{mol}$ photons $\mathrm{m}^{-2} \mathrm{~s}^{-1}$ for $30 \mathrm{~min}$. Interestingly, ETRII gradually decreased during the high-light treatment (Figure 1B). Meanwhile, the value of ETRI was higher than that of ETRII (Figure 1B), indicating the activation of CEF under high light. After this high-light treatment, the maximum photo-oxidizable
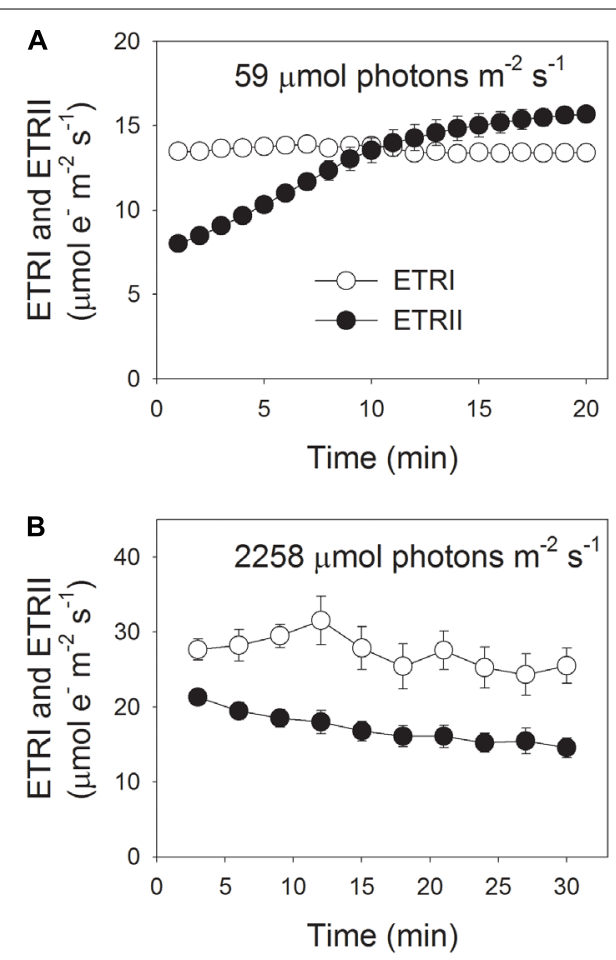

FIGURE 1 | Photosynthetic electron flow at low light (A) and high light (B). (A) Dark-acclimated leaves were exposed to a low light of $59 \mu \mathrm{mol}$ photons $\mathrm{m}^{-2} \mathrm{~s}^{-1}$ for $20 \mathrm{~min}$. (B) After illumination at $59 \mu \mathrm{mol}$ photons $\mathrm{m}^{-2} \mathrm{~s}^{-1}$ for $20 \mathrm{~min}$ to activate the electron sink in photosynthesis, leaves were exposed to a high light of $2258 \mu \mathrm{mol}$ photons $\mathrm{m}^{-2} \mathrm{~s}^{-1}$ for $30 \mathrm{~min}$. Values are means \pm SE $(n=5)$. 
P700 $\left(P_{\mathrm{m}}\right)$ was maintained stable (Figure 2A). By comparison, the maximum fluorescence intensity $\left(F_{\mathrm{m}}\right)$ decreased by $40 \%$ and the maximum quantum yield of PSII $\left(F_{\mathrm{V}} / F_{\mathrm{m}}\right)$ decreased from 0.80 to 0.65 (Figures $2 \mathrm{~B}, \mathrm{C}$ ). These results indicated the selective photoinhibition of PSII in leaves of Panax notoginseng under high-light stress.

Before high-light treatment, mature leaves were illuminated at a low light of $59 \mu \mathrm{mol}$ photons $\mathrm{m}^{-2} \mathrm{~s}^{-1}$ for $20 \mathrm{~min}$ to activate photosynthesis, and then values for ETRI, ETRII, $\mathrm{Y}(\mathrm{ND})$, and NPQ were recorded. After high-light treatment for $30 \mathrm{~min}$, values for ETRI, ETRII, Y(ND), and NPQ were recorded following a new acclimation phase of 20 min to low light. Before high-light treatment, values for ETRI and ETRII at $59 \mu \mathrm{mol}$ photons $\mathrm{m}^{-2} \mathrm{~s}^{-1}$ were 13.4 and $15.7 \mu \mathrm{mol}$ electrons $\mathrm{m}^{-2} \mathrm{~s}^{-1}$, respectively (Figures 3A,B). After high-light treatment,
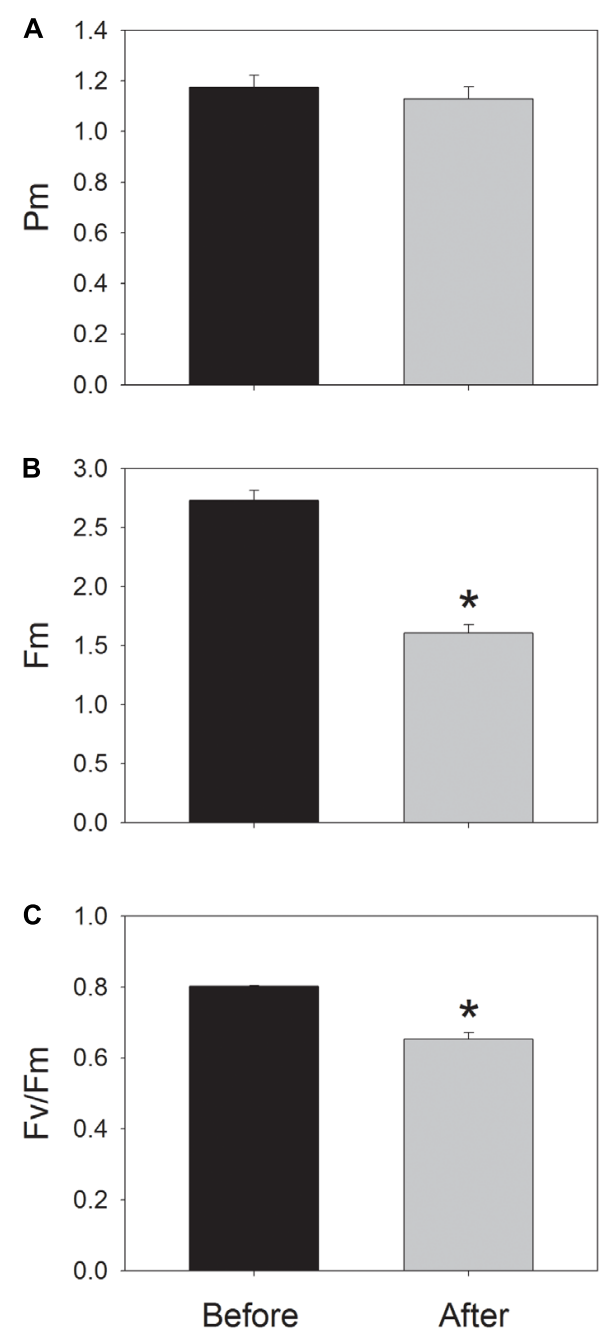

FIGURE 2 | Values for the maximum photo-oxidizable P700 $\left(P_{m}\right)(\mathbf{A})$, the maximum fluorescence intensity $\left(F_{m}\right)(\mathbf{B})$, and the maximum quantum yield of PSII $\left(F_{\mathrm{v}} / F_{\mathrm{m}}\right)(\mathbf{C})$ before and after treatment at $2258 \mu \mathrm{mol}$ photons $\mathrm{m}^{-2} \mathrm{~s}^{-1}$ for $30 \mathrm{~min}$. $P_{\mathrm{m}}$ and $F_{\mathrm{v}} / F_{\mathrm{m}}$ were measured after dark-acclimation for $30 \mathrm{~min}$. Values are means \pm SE $(n=5)$. Asterisk indicates a significant $(P<0.05)$ change after the high-light treatment.
ETRI and ETRII at low light were 12.8 and $11.2 \mu \mathrm{mol}$ electrons $\mathrm{m}^{-2} \mathrm{~s}^{-1}$, respectively (Figures 3A,B). The value of ETRII at $59 \mu \mathrm{mol}$ photons $\mathrm{m}^{-2} \mathrm{~s}^{-1}$ decreased by approximately $30 \%$ after photoinhibitory treatment, indicating the depression of ETRII upon moderate PSII photoinhibition. Before high-light treatment, the value of ETRI/ETRII ratio at $59 \mu \mathrm{mol}$ photons $\mathrm{m}^{-2} \mathrm{~s}^{-1}$ was 0.85 (Figure 3C). After photoinhibitory treatment, the ETRI/ETRII ratio significantly increased to 1.14 (Figure 3C). These results suggested the stimulation of CEF at low light upon PSII photoinhibition (Yamori et al., 2011, 2015).

After the high-light treatment, the steady-state value of $\mathrm{Y}(\mathrm{I})$ at $59 \mu \mathrm{mol}$ photons $\mathrm{m}^{-2} \mathrm{~s}^{-1}$ did not change (Figure 4A). Interestingly, $\mathrm{Y}(\mathrm{NA})$ significantly decreased from 0.36 to 0.23 (Figure 4B), and $\mathrm{Y}(\mathrm{ND})$ significantly increased from 0.1 to 0.25 (Figure 4C). These results indicated the change in redox state of PSI at low light after photoinhibitory treatment. Meanwhile, the value of NPQ changed slightly (Figure 4D). As a result,

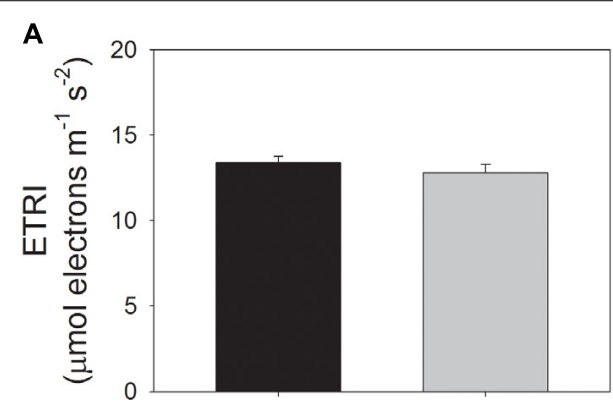

B

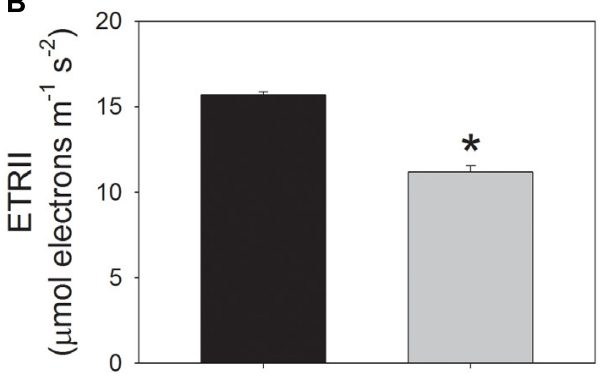

C

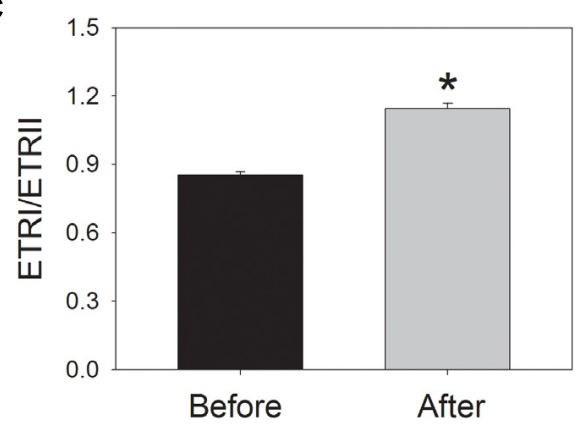

FIGURE 3 | Values for the electron flow through PSI (ETRI) (A), the electron flow from PSII (ETRII) (B), and the ETRI/ETRII ratio (C) at low light. Before and after high-light treatment, ETRI and ETRII were measured after illumination at $59 \mu \mathrm{mol}$ photons $\mathrm{m}^{-2} \mathrm{~s}^{-1}$ for $20 \mathrm{~min}$. Values are means $\pm \mathrm{SE}(n=5)$. Asterisk indicates a significant $(P<0.05)$ change after the high-light treatment. 

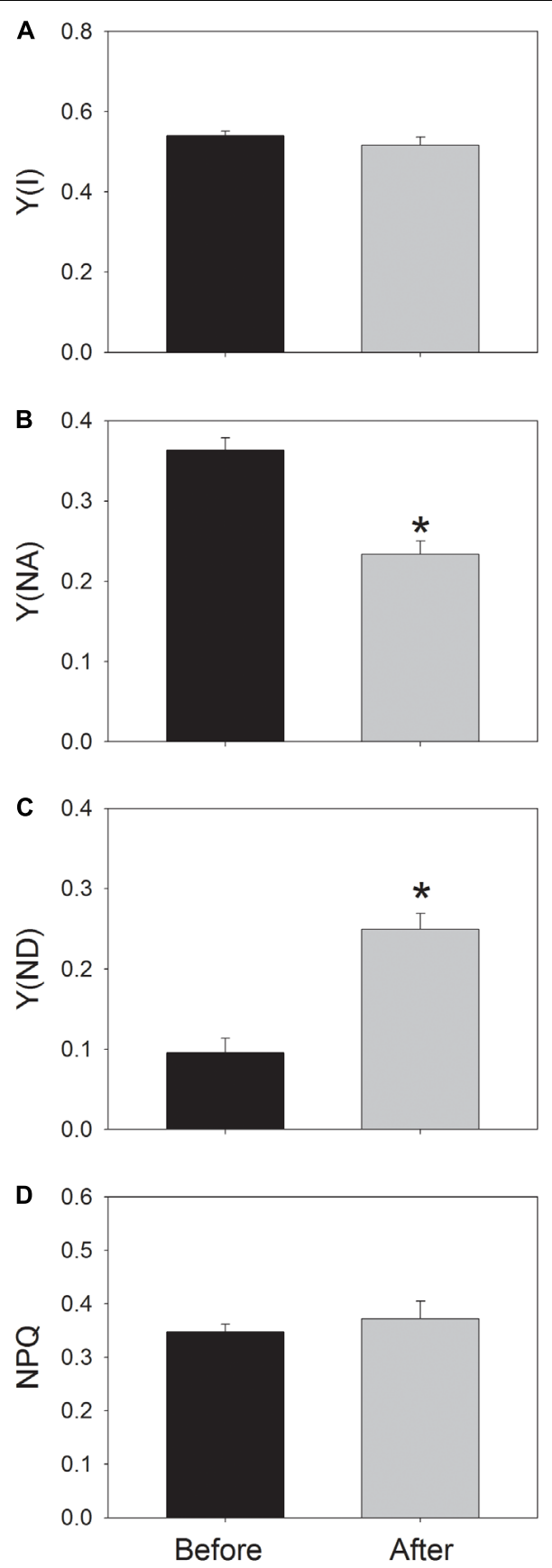

FIGURE 4 | Values for $Y($ (I) (A), Y(NA) (B), Y(ND) (C), and NPQ (D) at low light. Before and after high-light treatment, $Y(N D)$ and NPQ were measured after illumination at $59 \mu \mathrm{mol}$ photons $\mathrm{m}^{-2} \mathrm{~s}^{-1}$ for $20 \mathrm{~min}$. Values are means $\pm \mathrm{SE}$ $(n=5)$. Asterisk indicates a significant $(P<0.05)$ change after high-light treatment.

the moderate PSII photoinhibition had different effects on P700 oxidation ratio and NPQ at low light.

In order to clarify whether the increase in $\mathrm{Y}(\mathrm{ND})$ is caused by an increase in $p m f$, the electrochromic shift signals at $54 \mu \mathrm{mol}$ photons $\mathrm{m}^{-2} \mathrm{~s}^{-1}$ were determined after light acclimation for $20 \mathrm{~min}$ before and after the high-light treatment. Interestingly, the total pmf significantly decreased by $18 \%$ after high-light treatment (Figure 5A), but the $\Delta \mathrm{pH}$ level changed insignificantly (Figure 5B). Because the formation of pmf can be affected by the thylakoid proton conductivity, the proton conductivity $\left(g_{\mathrm{H}}{ }^{+}\right)$ of the thylakoid membrane at this low light was also examined before and after high-light treatment. The result showed that $g_{\mathrm{H}}+$ did not change with the high-light treatment (Figure 5C), suggesting that the high-light treatment hardly affected the activity of the chloroplast ATP synthase at low light. Because the decrease of $30 \%$ in ETRII was accompanied by a decrease of $18 \%$ in $p m f$, the stimulation of CEF at low light partly compensated for the formation of $p m f$ and $\Delta \mathrm{pH}$.

\section{DISCUSSION}

\section{The Role of CEF Stimulation at Low Light}

It has been indicated that CEF plays important roles in sustaining photosynthesis and plant growth (Yamori and Shikanai, 2016). Under high light, CEF is now thought to be essential for balancing the ATP/NADPH energy budget as well as for protecting PSI and PSII from damage (Munekage et al., 2002, 2004; Takahashi et al., 2009; Suorsa et al., 2012, 2016; Walker et al., 2014; Huang et al., 2015a, 2017b). At low light intensity, CEF plays an important role in optimizing photosynthetic $\mathrm{CO}_{2}$ assimilation probably via the supply of extra ATP (Yamori et al., 2011, 2015; Nishikawa et al., 2012; Huang et al., 2015a). The main role of CEF is modulated flexibly in response to light intensity. In particular, CEF mainly contributes to balancing the ATP/NADPH energy budget under subsaturating light intensities, but mainly protects photosynthetic apparatus against photoinhibition when exposed to saturating light intensities via acidification of the thylakoid lumen (Huang et al., 2015a). Interestingly, Huang et al. (2010) found that CEF was significantly stimulated at low light after chillinginduced photoinhibition of PSII and hypothesized that this CEF stimulation mainly enhanced the synthesis of ATP for the fast repair of PSII. However, more evidence was needed to support this hypothesis. In the present study, we observed that the value of ETRI/ETRII at low light significantly increased (Figure 3C), indicating the stimulation of CEF around PSI (Huang et al., 2011, 2012, 2017a,b; Yamori et al., 2011, 2015). Concomitantly, the amplitude of pmf decreased significantly and $\Delta \mathrm{pH}$ declined slightly. These results indicated that the stimulation of CEF did not trigger the $\Delta \mathrm{pH}$-dependent downregulation of photosynthetic electron transport. In the other words, this stimulation of CEF at low light mainly facilitated the synthesis of ATP.

Although the proton conductivity of the chloroplast ATP synthase was not affected by the high-light treatment, the smaller size of the pmf resulted in a decrease in the actual rate of ATP synthesis. The rates of PSII repair and photosynthetic $\mathrm{CO}_{2}$ assimilation at low light are mainly limited by the rate of ATP synthesis (Allakhverdiev et al., 2005; Yamori et al., 2011, 2015; Nishikawa et al., 2012). Because the ETRII significantly declined upon PSII photoinhibition, the rate of ATP synthesis via ETRII was remarkably reduced. In order to balance the 


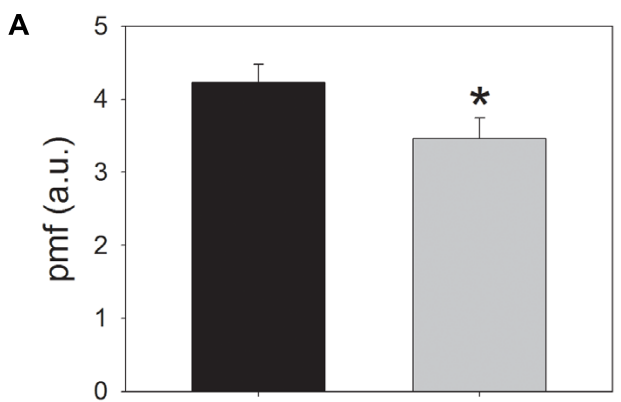

B

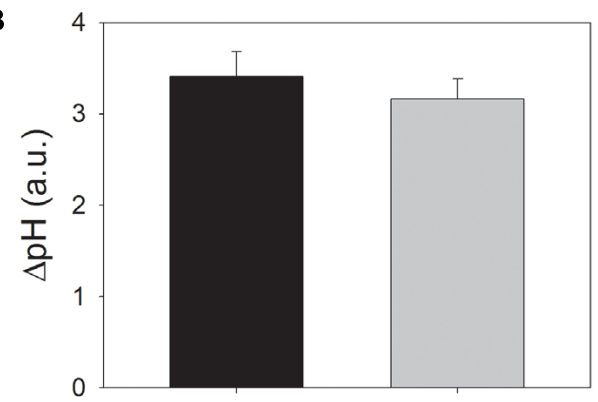

C

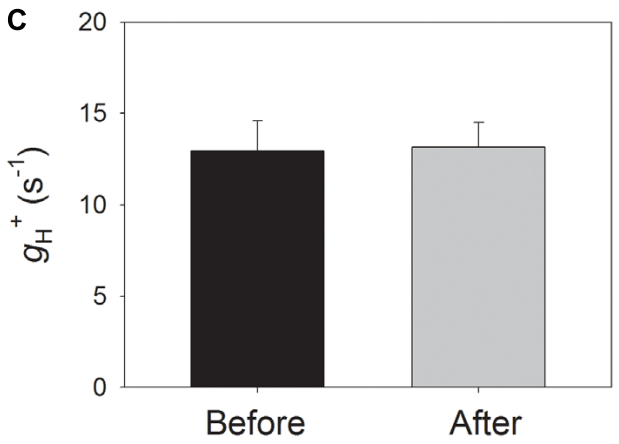

FIGURE $\mathbf{5}$ | Values for the total proton motive force (pmf) (A), the proton gradient $(\Delta \mathrm{pH})$ across the thylakoid membranes $\mathbf{( B )}$, and the proton conductivity $\left(\mathrm{gH}_{\mathrm{H}^{+}}\right)$of the thylakoid membrane (C) at low light. All parameters were measured after adaptation at $54 \mu \mathrm{mol}$ photons $\mathrm{m}^{-2} \mathrm{~s}^{-1}$ for $20 \mathrm{~min}$. Values are means \pm SE $(n=5)$. Asterisk indicates a significant $(P<0.05)$ change after high-light treatment.

ATP/NADPH ratio required by primary metabolism and the fast repair of PSII, a flexible mechanism is needed to provide extra ATP. Under this condition, ETRII decreased by $30 \%$ but the total pmf decreased by just $18 \%$. These results indicate that the stimulation of CEF compensated for the reduction in ETRII-dependent formation of pmf, enhancing the synthesis of ATP. The rapid repair of photodamaged PSII is dependent on ATP synthesis (Allakhverdiev et al., 2005). Acceleration of CEF around PSI increased the intracellular concentration of ATP, thus accelerating the rate of PSII repair in Synechocystis (Allakhverdiev et al., 2005). Taken together, we propose that the stimulation of CEF at low light plays an important role in the fast repair of PSII activity via an additional ATP synthesis.

\section{Change in P700 Oxidation Ratio at Low Light Is Not Related to pmf}

An interesting phenomenon is that the P700 oxidation ratio at low light significantly increased upon moderate PSII photoinhibition (Huang et al., 2010), which is also showed in the present study. Under conditions in which absorbed light is in excess of the requirements for photosynthesis, CEF-dependent generation of $\Delta \mathrm{pH}$ down-regulates the activity of Cyt $b_{6} / f$ complex activity and controls electron flow from PSII to PSI (Suorsa et al., 2012, 2016; Shikanai, 2014, 2016), optimizing the redox state of P700 in PSI and minimizing ROS production in PSI during photosynthesis. In pgr5-plants of A. thaliana, P700 is reduced by electrons in the light due to the loss of $p m f$ generation. However, in the pgr5 mutant accumulating flavodiiron protein, the level of pmf was also restored to the wild-type level, and thus P700 was oxidized as in the wild-type (Yamamoto et al., 2016). In chlorophyll $b$-deficient wheat mutant lines, insufficient thylakoid proton gradient leads to over-reduction of PSI acceptor side and thus PSI photoinhibition under high light or high temperature (Brestic et al., 2015, 2016). Recently, some studies reported that the increased activity of the chloroplast ATP synthase impaired the formation of pmf and caused the overreduction of photosynthetic electron transport chain, resulting in photodamage of PSI under high light and fluctuating light (Kanazawa et al., 2017; Takagi et al., 2017). These reports support the critical role of pmf in maintaining P700 optimally oxidized under excess light energy.

Our present results indicated that after the high-light treatment for $30 \mathrm{~min}$, the total pmf formed at this low light significantly declined and the formation of $\Delta \mathrm{pH}$ changed slightly (Figures 5A,B). Meanwhile, the P700 oxidation ratio significantly increased from 0.1 (before treatment) to 0.25 (after treatment) (Figure 4C). These results strongly indicate that this increase in P700 oxidation ratio cannot be explained by the change in $p m f$, which is largely different from the correlation between $\mathrm{Y}(\mathrm{ND})$ and pmf under high light (Yamamoto et al., 2016; Takagi et al., 2017). Therefore, the regulating effect of pmf on P700 oxidation ratio is minimal at low light but becomes particularly crucial under high light or fluctuating light. Under high light, a high value of $\mathrm{Y}(\mathrm{ND})$ is usually accompanied with a high value of NPQ (Munekage et al., 2002, 2004; Kono et al., 2014; Zivcak et al., 2015), due to the increase in pmf. The impairment of $\Delta \mathrm{pH}$ formation leads to decreased levels of $\mathrm{Y}(\mathrm{ND})$ and NPQ under high light (Suorsa et al., 2012; Kono et al., 2014; Yamamoto et al., 2016; Kanazawa et al., 2017). The induction of NPQ at low light is mainly determined by the level of lumen acidification. After high-light treatment, the extent of lumen acidification did not change (Figure 5B), and NPQ remained stable (Figure 4D). By comparison, $\mathrm{Y}(\mathrm{ND})$ significantly increased. These results indicate that at low light, Y(ND) and NPQ were controlled by different regulatory mechanisms.

\section{ETRII Controls P700 Oxidation Ratio at Low Light}

PSI becomes over-reduced only when electron flow from PSII exceeds the capacity of PSI electron acceptors to cope with 
the electrons. When electron flow to PSI is limited, PSI is extremely tolerant against light stress in the pgr5 plants of A. thaliana (Suorsa et al., 2012, 2016; Tikkanen et al., 2014). For the shade-establishing plant Psychotria rubra, PSI activity was insusceptible to high-light stress in the presence of DCMU (Huang et al., 2016c). In chilled leaves of tobacco, moderate PSII photoinhibition allowed the maintenance of P700 optimally oxidized and then protected PSI activity against further photodamage (Huang et al., 2016a). These results indicated the important role of ETRII in controlling the redox state of PSI under high light or chilling-light stresses. Our present results showed that the moderate PSII photoinhibition led to a significant depression of ETRII at low light. Meanwhile, the PSI activity and the activity of the chloroplast ATP synthase changed slightly, and the total pmf and $\Delta \mathrm{pH}$ did not increase. As a result, the reduction in ETRII was not caused by the $\Delta \mathrm{pH}$-dependent photosynthetic control via Cyt $b_{6} / f$ complex, but was probably induced by the decrease in PSII activity (Tikkanen et al., 2014). The decreased supply of electrons from PSII to PSI led to the increased level of $\mathrm{P} 700$ oxidation. Thus, the P700 oxidation ratio at low light was largely controlled by ETRII.

\section{CONCLUSION}

We found that the selective photoinhibition of PSII induced a stimulation of CEF and an increase in P700 oxidation ratio

\section{REFERENCES}

Allakhverdiev, S. I., Nishiyama, Y., Takahashi, S., Miyairi, S., Suzuki, I., and Murata, N. (2005). Systematic analysis of the relation of electron transport and ATP synthesis to the photodamage and repair of photosystem II in Synechocystis. Plant Physiol. 137, 263-273. doi: 10.1104/pp.104.05 4478

Aro, E. M., Virgin, I., and Andersson, B. (1993). Photoinhibition of photosystem II. Inactivation, protein damage and turnover. Biochim. Biophys. Acta 1143, 113-134. doi: 10.1016/0005-2728(93)90134-2

Barth, C., Krause, G. H., and Winter, K. (2001). Responses of photosystem I compared with photosystem II to high-light stress in tropical shade and sun leaves. Plant Cell Environ. 24, 163-176. doi: 10.1111/j.1365-3040.2001.00673.x

Brestic, M., Zivcak, M., Kunderlikova, K., and Allakhverdiev, S. I. (2016). High temperature specifically affects the photoprotective responses of chlorophyll b-deficient wheat mutant lines. Photosynth. Res. 130, 251-266. doi: 10.1007/ s11120-016-0249-7

Brestic, M., Zivcak, M., Kunderlikova, K., Sytar, O., Shao, H., Kalaji, H. M., et al. (2015). Low PSI content limits the photoprotection of PSI and PSII in early growth stages of chlorophyll b-deficient wheat mutant lines. Photosynth. Res. 125, 151-166. doi: 10.1007/s11120-015-0093-1

Burrows, P. A., Sazanov, L. A., Svab, Z., Maliga, P., and Nixon, P. J. (1998). Identification of a functional respiratory complex in chloroplasts through analysis of tobacco mutants containing disrupted plastid ndh genes. EMBO J. 17, 868-876. doi: 10.1093/emboj/17. 4.868

Cruz, J. A., Avenson, T. J., Kanazawa, A., Takizawa, K., Edwards, G. E., and Kramer, D. M. (2005). Plasticity in light reactions of photosynthesis for energy production and photoprotection. J. Exp. Bot. 56, 395-406. doi: 10.1093/jxb/ eri022

Genty, B., Briantais, J. M., and Baker, N. R. (1989). The relationship between the quantum yield of photosynthetic electron transport and quenching of chlorophyll fluorescence. Biochim. Biophys. Acta 990, 87-92. doi: 10.1016/ S0304-4165(89)80016-9 at low light. The stimulation of CEF did not trigger the $\Delta \mathrm{pH}$-dependent down-regulation of photosynthetic electron transport. As a result, this stimulation of CEF at low light mainly facilitated the synthesis of ATP, which is essential for the rapid repair of photodamaged PSII. The increase in P700 oxidation ratio could not be explained by the change in $\Delta \mathrm{pH}$ dependent photosynthetic control at the Cyt $b_{6} / f$ complex, but was primarily caused by the decreased supply of electrons from PSII to PSI.

\section{AUTHOR CONTRIBUTIONS}

$\mathrm{WH}, \mathrm{S}-\mathrm{BZ}$, and TL conceived and designed the research. WH and Y-JY conducted the experiments. WH, Y-JY, S-BZ, and TL analyzed the data. WH wrote the first draft of the manuscript, which was intensively edited by all the authors.

\section{FUNDING}

This study was supported by the National Natural Science Foundation of China (31670343), Youth Innovation Promotion Association of the Chinese Academy of Sciences (2016347), and the Major Science and Technique Programs in Yunnan Province (2016ZF001).

Grieco, M., Tikkanen, M., Paakkarinen, V., Kangasjarvi, S., and Aro, E. M. (2012). Steady-state phosphorylation of light-harvesting complex II proteins preserves photosystem I under fluctuating white light. Plant Physiol. 160, 1896-1910. doi: 10.1104/pp.112.206466

He, J., and Chow, W. S. (2003). The rate coefficient of repair of photosystem II after photoinactivation. Physiol. Plant. 118, 297-304. doi: 10.1034/j.1399-3054.2003. 00107.x

Huang, W., Yang, S. J., Zhang, S. B., Zhang, J. L., and Cao, K. F. (2012). Cyclic electron flow plays an important role in photoprotection for the resurrection plant Paraboea rufescens under drought stress. Planta 235, 819-828. doi: 10.1007/s00425-011-1544-3

Huang, W., Yang, Y. J., Hu, H., and Zhang, S. B. (2016a). Moderate photoinhibition of photosystem II protects photosystem I from photodamage at chilling stress in tobacco leaves. Front. Plant Sci. 7:182. doi: 10.3389/fpls.2016. 00182

Huang, W., Yang, Y. J., Hu, H., and Zhang, S. B. (2016b). Responses of photosystem I compared with photosystem II to fluctuating light in the shade-establishing tropical tree species Psychotria henryi. Front. Plant Sci. 7:1549. doi: 10.3389/ fpls.2016.01549

Huang, W., Yang, Y. J., Zhang, J. L., Hu, H., and Zhang, S. B. (2016c). PSI photoinhibition is more related to electron transfer from PSII to PSI rather than PSI redox state in Psychotria rubra. Photosynth. Res. 129, 85-92. doi: 10.1007/s11120-016-0275-5

Huang, W., Yang, Y. J., Zhang, J. L., Hu, H., and Zhang, S. B. (2017a). Superoxide generated in the chloroplast stroma causes photoinhibition of photosystem I in the shade-establishing tree species Psychotria henryi. Photosynth. Res. 132, 293-303. doi: 10.1007/s11120-017-0389-4

Huang, W., Yang, Y. J., and Zhang, S. B. (2017b). Specific roles of cyclic electron flow around photosystem I in photosynthetic regulation in immature and mature leaves. J. Plant Physiol. 209, 76-83. doi: 10.1016/j.jplph.2016. 11.013

Huang, W., Zhang, S. B., and Cao, K. F. (2010). Stimulation of cyclic electron flow during recovery after chilling-induced photoinhibition of PSII. Plant Cell Physiol. 51, 1922-1928. doi: 10.1093/pcp/pcq144 
Huang, W., Zhang, S. B., and Cao, K. F. (2011). Cyclic electron flow plays an important role in photoprotection of tropical trees illuminated at temporal chilling temperature. Plant Cell Physiol. 52, 297-305. doi: 10.1093/pcp/ pcq166

Huang, W., Yang, Y. J., Hu, H., and Zhang, S. B. (2015a). Different roles of cyclic electron flow around photosystem I under sub-saturating and saturating light intensities in tobacco leaves. Front. Plant Sci. 6:923. doi: 10.3389/fpls.2015. 00923

Huang, W., Zhang, S. B., Zhang, J. L., and Hu, H. (2015b). Photoinhibition of photosystem I under high light in the shade-established tropical tree species Psychotria rubra. Front. Plant Sci. 6:801. doi: 10.3389/fpls.2015.00801

Joliot, P., and Joliot, A. (2002). Cyclic electron transfer in plant leaf. Proc. Natl. Acad. Sci. U.S.A. 99, 10209-10214. doi: 10.1073/pnas.102306999

Joliot, P., and Joliot, A. (2005). Quantification of cyclic and linear flows in plants. Proc. Natl. Acad. Sci. U.S.A. 102, 4913-4918. doi: 10.1073/pnas.0501268102

Kanazawa, A., Ostendorf, E., Kohzuma, K., Hoh, D., Strand, D. D., SatoCruz, M., et al. (2017). Chloroplast ATP synthase modulation of the thylakoid proton motive force: implications for photosystem I and photosystem II photoprotection. Front. Plant Sci. 8:719. doi: 10.3389/fpls.2017. 00719

Kitao, M., Lei, T. T., Koike, T., Tobita, H., and Muruyama, Y. (2000). Susceptibility to photoinhibition of three deciduous broadleaf tree species with different successional traits raised under various light regimes. Plant Cell Environ. 23, 81-89. doi: 10.1046/j.1365-3040.2000.00528.x

Klughammer, C., and Schreiber, U. (2008). Saturation pulse method for assessment of energy conversion in PSI. PAM Appl. Notes 1, 11-14.

Kono, M., Noguchi, K., and Terashima, I. (2014). Roles of the cyclic electron flow around PSI (CEF-PSI) and $\mathrm{O}_{2}$-dependent alternative pathways in regulation of the photosynthetic electron flow in short-term fluctuating light in Arabidopsis thaliana. Plant Cell Physiol. 55, 990-1004. doi: 10.1093/pcp/pcu033

Krall, J. P., and Edwards, G. E. (1992). Relationship between photosystem II activity and $\mathrm{CO}_{2}$ fixation in leaves. Physiol. Plant. 86, 180-187. doi: 10.1111/j.13993054.1992.tb01328.x

Krause, G. H., Grube, E., Koroleva, O. Y., Barth, C., and Winter, K. (2004). Do mature shade leaves of tropical tree seedlings acclimate to high sunlight and UV radiation? Funct. Plant Biol. 31, 743-756. doi: 10.1071/FP03239

Lunde, C., Jensen, P. E., Rosgaard, L., Haldrup, A., Gilpin, M. J., and Scheller, H. V. (2003). Plants impaired in state transitions can to a large degree compensate for their defect. Plant Cell Physiol. 44, 44-54. doi: 10.1093/pcp/pcg012

Makino, A., Miyake, C., and Yokota, A. (2002). Physiological functions of the water-water cycle (Mehler reaction) and the cyclic electron flow around PSI in rice leaves. Plant Cell Physiol. 43, 1017-1026. doi: 10.1093/pcp/ pcf124

Munekage, Y., Hashimoto, M., Miyake, C., Tomizawa, K. I., Endo, T., Tasaka, M., et al. (2004). Cyclic electron flow around photosystem I is essential for photosynthesis. Nature 429, 579-582. doi: 10.1038/nature02598

Munekage, Y., Hojo, M., Meurer, J., Endo, T., Tasaka, M., and Shikanai, T. (2002). PGR5 is involved in cyclic electron flow around photosystem I and is essential for photoprotection in Arabidopsis. Cell 110, 361-371. doi: 10.1016/S00928674(02)00867-X

Nishikawa, Y., Yamamoto, H., Okegawa, Y., Wada, S., Sato, N., Taira, Y., et al. (2012). PGR5-dependent cyclic electron transport around PSI contributes to the redox homeostasis in chloroplasts rather than $\mathrm{CO}_{2}$ fixation and biomass production in rice. Plant Cell Physiol. 53, 2117-2126. doi: 10.1093/pcp/ pcs153

Sacksteder, C. A., Jacoby, M. E., and Kramer, D. M. (2001). A portable, nonfocusing optics spectrometer (NoFOSpec) for measurements of steady-state absorbance changes in intact plants. Photosynth. Res. 70, 231-240. doi: 10.1023/ A:1017906626288

Sacksteder, C. A., and Kramer, D. M. (2000). Dark interval relaxation kinetics of absorbance changes as a quantitative probe of steady-state electron transfer. Photosynth. Res. 66, 145-158. doi: 10.1023/A:1010785912271

Shikanai, T. (2007). Cyclic electron transport around photosystem I: genetic approaches. Annu. Rev. Plant Biol. 58, 199-217. doi: 10.1146/annurev.arplant. 58.091406 .110525

Shikanai, T. (2014). Central role of cyclic electron transport around photosystem I in the regulation of photosynthesis. Curr. Opin. Biotech. 26, 25-30. doi: 10.1016/j.copbio.2013.08.012
Shikanai, T. (2016). Regulatory network of proton motive force: contribution of cyclic electron transport around photosystem I. Photosynth. Res. 129, 253-260. doi: 10.1007/s11120-016-0227-0

Shikanai, T., Endo, T., Hashimoto, T., Yamada, Y., Asada, K., and Yokota, A. (1998). Directed disruption of the tobacco ndhB gene impairs cyclic electron flow around photosystem I. Proc. Natl. Acad. Sci. U.S.A. 95, 9705-9709. doi: $10.1073 /$ pnas. 95.16 .9705

Shikanai, T., and Yamamoto, H. (2017). Contribution of cyclic and pseudo-cyclic electron transport to the formation of proton motive force in chloroplasts. Mol. Plant. 10, 20-29. doi: 10.1016/j.molp.2016.08.004

Sugimoto, K., Okegawa, Y., Tohri, A., Long, T. A., Covert, S. F., Hisabori, T., et al. (2013). A single amino acid alteration in PGR5 confers resistance to antimycin A in cyclic electron transport around PSI. Plant Cell Physiol. 54, 1525-1534. doi: $10.1093 / p c p / p c t 098$

Suorsa, M., Jarvi, S., Grieco, M., Nurmi, M., Pietrzykowska, M., Rantala, M., et al. (2012). PROTON GRADIENT REGULATION5 is essential for proper acclimation of Arabidopsis photosystem I to naturally and artificially fluctuating light conditions. Plant Cell 24, 2934-2948. doi: 10.1105/tpc.112.09 7162

Suorsa, M., Rossi, F., Tadini, L., Labs, M., Colombo, M., Jahns, P., et al. (2016). PGR5-PGRL1-dependent cyclic electron transport modulates linear electron transport rate in Arabidopsis thaliana. Mol. Plant 9, 271-288. doi: 10.1016/j. molp.2015.12.001

Takagi, D., Amako, K., Hashiguchi, M., Fukaki, H., Ishizaki, K., Goh, T., et al. (2017). Chloroplastic ATP synthase builds up proton motive force for preventing reactive oxygen species production in photosystem I. Plant J. 91, 306-324. doi: 10.1111/tpj.13566

Takahashi, S., Milward, S. E., Fan, D. Y., Chow, W. S., and Badger, M. R. (2009). How does cyclic electron flow alleviate photoinhibition in Arabidopsis? Plant Physiol. 149, 1560-1567. doi: 10.1104/pp.108.134122

Takizawa, K., Kanazawa, A., and Kramer, D. M. (2008). Depletion of stromal Pi induces high 'energy-dependent' antenna exciton quenching $(\mathrm{qE})$ by decreasing proton conductivity at CFO-CF1 ATP synthase. Plant Cell Environ. 31, 235-243. doi: 10.1111/j.1365-3040.2007.01753.x

Tikkanen, M., and Aro, E. M. (2014). Integrative regulatory network of plant thylakoid energy transduction. Trends. Plant Sci. 19, 10-17. doi: 10.1016/j. tplants.2013.09.003

Tikkanen, M., Mekala, N. R., and Aro, E. M. (2014). Photosystem II photoinhibition-repair cycle protects Photosystem I from irreversible damage. Biochim. Biophys. Acta 1837, 210-215. doi: 10.1016/j.bbabio.2013.10.001

Tikkanen, M., Piippo, M., Suorsa, M., Sirpio, S., Mulo, P., Vainonen, J., et al. (2006). State transitions revisited-a buffering system for dynamic low light acclimation of Arabidopsis. Plant Mol. Biol. 62, 779-793. doi: 10.1007/s11103-0069088-9

Tikkanen, M., Rantala, S., Grieco, M., and Aro, E. M. (2017). Comparative analysis of mutant plants impaired in the main regulatory mechanisms of photosynthetic light reactions - From biophysical measurements to molecular mechanisms. Plant Physiol. Biochem. 112, 290-301. doi: 10.1016/j.plaphy.2017. 01.014

Walker, B. J., Strand, D. D., Kramer, D. M., and Cousins, A. B. (2014). The response of cyclic electron flow around photosystem I to changes in photorespiration and nitrate assimilation. Plant Physiol. 165, 453-462. doi: 10.1104/pp.114.23 8238

Yamamoto, H., Takahashi, S., Badger, M. R., and Shikanai, T. (2016). Artificial remodeling of alternative electron flow by flavodiiron proteins in Arabidopsis. Nat. Plants 2:16012. doi: 10.1038/nplants.2016.12

Yamori, W., Makino, A., and Shikanai, T. (2016). A physiological role of cyclic electron transport around photosystem I in sustaining photosynthesis under fluctuating light in rice. Sci. Rep. 6:20147. doi: 10.1038/srep20147

Yamori, W., Sakata, N., Suzuki, Y., Shikanai, T., and Maniko, A. (2011). Cyclic electron flow around photosystem I via chloroplast NAD(P)H dehydrogenase $(\mathrm{NDH})$ complex performs a significant physiological role during photosynthesis and plant growth at low temperature in rice. Plant J. 68, 966-976. doi: 10.1111/j.1365-313X.2011.04747.x

Yamori, W., and Shikanai, T. (2016). Physiological functions of cyclic electron transport around photosystem I in sustaining photosynthesis and plant growth. Annu. Rev. Plant Biol. 67, 81-106. doi: 10.1146/annurev-arplant-043015112002 
Yamori, W., Shikanai, T., and Makino, A. (2015). Photosystem I cyclic electron flow via chloroplast NADH dehydrogenase-like complex performs a physiological role for photosynthesis at low light. Sci. Rep. 5:13908. doi: 10.1038/srep 13908

Zhang, S. P., and Scheller, H. V. (2004). Photoinhibition of photosystem I at chilling temperature and subsequent recovery in Arabidopsis thaliana. Plant Cell Physiol. 45, 1595-1602. doi: 10.1093/pcp/pch180

Zivcak, M., Brestic, M., Kunderlikova, K., Sytar, O., and Allakhverdiev, S. I. (2015). Repetitive light pulse-induced photoinhibition of photosystem I severely affects $\mathrm{CO}_{2}$ assimilation and photoprotection in wheat leaves. Photosynth. Res. 126, 449-463. doi: 10.1007/s11120-015-0121-1
Conflict of Interest Statement: The authors declare that the research was conducted in the absence of any commercial or financial relationships that could be construed as a potential conflict of interest.

Copyright (c) 2018 Huang, Yang, Zhang and Liu. This is an open-access article distributed under the terms of the Creative Commons Attribution License (CC BY). The use, distribution or reproduction in other forums is permitted, provided the original author(s) and the copyright owner are credited and that the original publication in this journal is cited, in accordance with accepted academic practice. No use, distribution or reproduction is permitted which does not comply with these terms. 